\title{
Adaptive Transmission with Partial Channel Information in Spatially Correlated MIMO Channels
}

\author{
Jae-Yun Ko and Yong-Hwan Lee \\ School of Electrical Engineering and INMC, Seoul National University \\ Kwanak P. O. Box 34, Seoul, 151-600 Korea
}

\begin{abstract}
We propose a new adaptive multiple-input multipleoutput (MIMO) transmission scheme that can work with partial channel information. Utilizing the information on dominant eigendimensions of the channel correlation matrix, the proposed scheme reduces the amount of channel information required for adaptive transmission without noticeable performance degradation. It is analytically shown that the proposed scheme can minimize the performance loss by properly choosing the number of eigen-dimensions of the channel correlation matrix. Simulation results show that the proposed scheme is quite applicable to practical systems where quantized channel information is utilized.
\end{abstract}

\section{INTRODUCTION}

In multiple-input multiple-output (MIMO) systems, channel information plays an important role in achieving high system capacity. When the transmitter has full channel knowledge, it can achieve the channel capacity by making the use of singular value decomposition (SVD) and water-filling power allocation technique [1] (called adaptive transmission in this paper). The MIMO channel is usually expressed by a number of complex values and the channel information needs to be updated periodically at the transmitter due to the nature of time-varying properties. In practice, the channel information can be informed from the receiver, which may require a large feedback signaling overhead. Efficient quantization methods have been developed to reduce the feedback signaling burden [2], [3]. However, large quantization noise may not be avoidable especially when the number of transmit antennas is large.

Meanwhile, when the transmitter is aware of the correlation of the channel, the performance can be improved by allocating more power to dominant eigenmodes [4], [5]. Since the correlation can be assumed unchanged during the time interval much longer than the duration of fading [6], it can be informed to the transmitter without significant increase of feedback signaling overhead. However, the use of channel correlation information is not sufficient for the maximization of the capacity [7], resulting in a large capacity loss compared to the use of full channel information especially when the channel is partially correlated.

In this paper, we consider the use of partial channel information for the adaptive transmission. By utilizing dominant eigen-dimensions of the channel correlation matrix, the system can have an effective channel with a reduced dimension, enabling to reduce the amount of feedback signaling for the adaptive transmission. It is analytically shown that the proposed scheme can provide a capacity almost the same as that utilizing full channel information when dominant eigen-dimensions of the channel correlation matrix are chosen to contain most of the channel energy. It is also shown that the proposed scheme is quite applicable to practical systems with a finite-rate feedback channel.

This paper is organized as follows. Section II describes the system model in consideration. The proposed scheme is described in Section III and the performance is analyzed in Section IV. In Section V, we verify the performance by computer simulation. Finally, Section VI concludes the paper.

\section{System Model}

Consider a MIMO system that utilizes $n_{\mathrm{t}}$ transmit antennas and $n_{\mathrm{r}}$ receive antennas. The received signal can be represented by

$$
\mathbf{y}=\mathbf{H x}+\mathbf{n}
$$

where $\mathbf{H}$ denotes the $\left(n_{\mathrm{r}} \times n_{\mathrm{t}}\right)$-dimensional channel matrix whose elements are random variables with zero mean and unit variance, $\mathbf{x}$ is the $\left(n_{\mathrm{t}} \times 1\right)$-dimensional transmit signal vector with a power of $P$ and $\mathbf{n}$ is $\left(n_{\mathrm{r}} \times 1\right)$-dimensional additive white Gaussian noise (AWGN) whose elements have unit variance.

We define the channel correlation matrix of transmitter by

$$
\mathbf{R}_{\mathrm{t}} \triangleq E\left\{\mathbf{H}^{*} \mathbf{H}\right\} / n_{\mathrm{r}}
$$

where the superscript $*$ denotes the conjugate transpose. The correlation matrix can be decomposed as

$$
\mathbf{R}_{\mathrm{t}}=\mathbf{Q} \boldsymbol{\Sigma}^{2} \mathbf{Q}^{*}
$$

where $\mathbf{Q}=\left[\mathbf{q}_{1} \ldots \mathbf{q}_{n_{\mathrm{t}}}\right]$ is an $\left(n_{\mathrm{t}} \times n_{\mathrm{t}}\right)$-dimensional unitary matrix and $\boldsymbol{\Sigma}$ is an $\left(n_{\mathrm{t}} \times n_{\mathrm{t}}\right)$-dimensional diagonal matrix with ordered diagonal elements $\sigma_{1} \geq \cdots \geq \sigma_{n_{t}}$ where $\sum_{i=1}^{n_{i}} \sigma_{i}^{2}=n_{\mathrm{t}}$. Then the channel matrix can be represented as [4]

$$
\mathbf{H}=\tilde{\mathbf{H}} \mathbf{R}_{\mathrm{t}}^{1 / 2}
$$

where $\tilde{\mathbf{H}}$ is an $\left(n_{\mathrm{r}} \times n_{\mathrm{t}}\right)$-dimensional matrix whose columns 


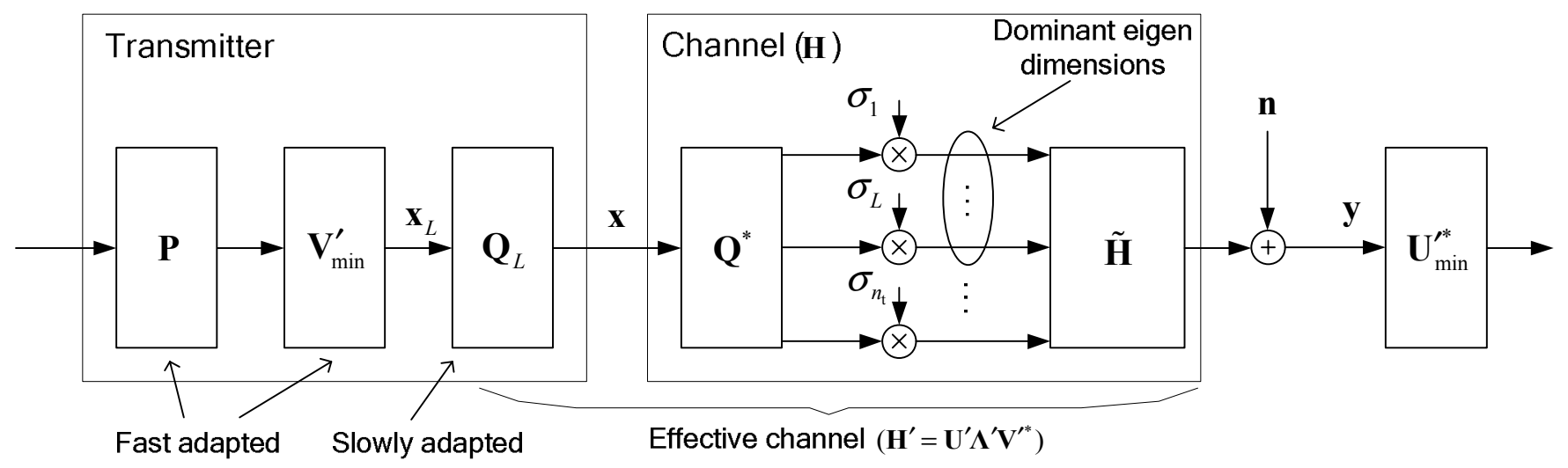

Fig. 1. Block diagram of the proposed partial adaptive transmission scheme.

are independent of each other and have the same expected norm value, i.e.,

$$
E\left[\tilde{\mathbf{h}}_{i}^{*} \tilde{\mathbf{h}}_{j}\right]= \begin{cases}0, & i \neq j \\ n_{\mathrm{r}}, & i=j .\end{cases}
$$

Here, $\tilde{\mathbf{h}}_{i}$ denotes the $i$-th column of $\tilde{\mathbf{H}}$.

\section{ADAPTIVE MIMO TRANSMISSION TECHNIQUES}

\section{A. Full Adaptive Transmission Scheme [1]}

The capacity of the MIMO system given by

$$
C=\log _{2} \operatorname{det}\left(\mathbf{I}+\mathbf{H} \mathbf{K}_{x} \mathbf{H}^{*}\right)
$$

can be maximized by optimizing the covariance matrix $\mathbf{K}_{x} \triangleq E\left\{\mathbf{x} \mathbf{x}^{*}\right\}$ of the transmit signal. The optimum covariance matrix is directly related to the channel matrix which can be decomposed as

$$
\mathbf{H}=\mathbf{U} \boldsymbol{\Lambda} \mathbf{V}^{*}
$$

where $\mathbf{U}=\left[\mathbf{u}_{1} \cdots \mathbf{u}_{n_{\mathrm{r}}}\right]$ and $\mathbf{V}=\left[\mathbf{v}_{1} \cdots \mathbf{v}_{n_{\mathrm{t}}}\right]$ are $\left(n_{\mathrm{r}} \times n_{\mathrm{r}}\right)$ and $\left(n_{\mathrm{t}} \times n_{\mathrm{t}}\right)$-dimensional unitary matrices, respectively, and $\boldsymbol{\Lambda}$ is an $\left(n_{\mathrm{r}} \times n_{\mathrm{t}}\right)$-dimensional rectangular matrix that has nonnegative real and ordered diagonal elements $\lambda_{1} \geq \cdots \geq \lambda_{n_{\min }}$ and zero off-diagonal elements, where $n_{\min }=\min \left(n_{\mathrm{t}}, n_{\mathrm{r}}\right)$.

The optimum $\mathbf{K}_{x}$ can be determined by [1]

$$
\mathbf{K}_{x}=\mathbf{V P V}^{*}
$$

where $\quad \mathbf{P}=\operatorname{diag}\left\{P_{1}, \ldots, P_{n_{\mathrm{t}}}\right\} \quad$ is an $\left(n_{\mathrm{t}} \times n_{\mathrm{t}}\right)$-dimensional diagonal matrix whose first $n_{\min }$ diagonal elements are obtained by water-filling power allocation along $\left\{\lambda_{i}, i=1, \ldots, n_{\min }\right\} \quad[1]$ and other elements are set to zero. Thus, the transmitter needs information on $\left(n_{\mathrm{t}} \times n_{\text {min }}\right)$-dimensional precoding matrix $\mathbf{V}_{\text {min }}=\left[\mathbf{v}_{1} \cdots \mathbf{v}_{n_{\min }}\right]$ in addition to the corresponding transmit power allocation $\left\{P_{i}, i=1, \ldots, n_{\min }\right\}$. The capacity of the full adaptive transmission scheme can be represented as

$$
C_{\text {full }}=\sum_{i=1}^{n_{\min }} \log _{2}\left(1+\lambda_{i}^{2} P_{i}\right)
$$

\section{B. Proposed Partial Adaptive Transmission Scheme}

Fig. 1 depicts a block diagram of the proposed scheme where only $L$ dominant eigen-dimensions of the channel correlation matrix (called virtual antennas in this paper) are considered. Since each eigen-dimension is represented by a column of $\mathbf{Q}$ [4], the $L$ dominant virtual antennas can be chosen by multiplying $\mathbf{Q}_{I}=\left[\mathbf{q}_{1} \ldots \mathbf{q}_{L}\right]$ as shown in Fig. 1 .

Letting $\mathbf{H}^{\prime} \triangleq \mathbf{H Q}_{L}$, the MIMO system with a reduced dimension can be represented as

$$
\mathbf{y}=\mathbf{H}^{\prime} \mathbf{x}_{L}+\mathbf{n}
$$

Since the reduced system has $L\left(\leq n_{\mathrm{t}}\right)$ virtual transmit antennas, further adaptive transmission procedure can be carried out using the effective channel matrix with a reduced dimension.

Using $\mathbf{H}=\tilde{\mathbf{H}} \mathbf{R}_{\mathrm{t}}^{1 / 2}$ and $\mathbf{R}_{\mathrm{t}}^{1 / 2}=\mathbf{Q} \boldsymbol{\Sigma} \mathbf{Q}^{*}$, the $\left(n_{\mathrm{r}} \times L\right)-$ dimensional effective channel $\mathbf{H}^{\prime}$ can be represented as

$$
\mathbf{H}^{\prime}=\tilde{\mathbf{H}} \mathbf{R}_{\mathrm{t}}^{1 / 2} \mathbf{Q}_{L}=\tilde{\mathbf{H}} \mathbf{Q} \boldsymbol{\Sigma} \mathbf{Q}^{*} \mathbf{Q}_{L}=\tilde{\mathbf{H}}^{\prime} \boldsymbol{\Sigma}_{L}
$$

where $\tilde{\mathbf{H}}^{\prime} \triangleq \tilde{\mathbf{H}} \mathbf{Q}$ has the same distribution as $\tilde{\mathbf{H}}$, and $\boldsymbol{\Sigma}_{L}$ is an $\left(n_{\mathrm{t}} \times L\right)$-dimensional matrix whose diagonal elements are the same as the first $L$ diagonal elements of $\boldsymbol{\Sigma}$ and whose offdiagonal elements are zero. Note that each diagonal element of $\Sigma$ represents the average channel gain from the corresponding virtual antenna.

The dimension-reduced MIMO channel can be decomposed as

$$
\mathbf{H}^{\prime}=\mathbf{U}^{\prime} \Lambda^{\prime} \mathbf{V}^{\prime *}
$$

where $\mathbf{U}^{\prime}$ and $\mathbf{V}^{\prime}$ are $\left(n_{\mathrm{r}} \times n_{\mathrm{r}}\right)$ and $(L \times L)$-dimensional unitary matrices, respectively, and $\boldsymbol{\Lambda}^{\prime}$ is an $\left(n_{\mathrm{r}} \times L\right)$ - 
dimensional rectangular matrix that has non-negative real ordered diagonal elements $\lambda_{1}^{\prime} \geq \cdots \geq \lambda_{n_{\min }^{\prime}}^{\prime}$ and zero offdiagonal elements, where $n_{\min }^{\prime}=\min \left(L, n_{\mathrm{r}}\right)$. For a given $\tilde{\mathbf{H}}^{\prime}$, the capacity of the dimension-reduced system can be maximized by optimizing the covariance matrix $\mathbf{K}_{x_{I}} \triangleq E\left\{\mathbf{x}_{L} \mathbf{x}_{L}^{*}\right\}$ using $\left(L \times n_{\text {min }}^{\prime}\right)$-dimensional precoding matrix $\mathbf{V}_{\min }^{\prime}$ which is obtained by the first $n_{\text {min }}^{\prime}$ columns of $\mathbf{V}^{\prime}$ and the corresponding transmit power $P_{i}^{\prime}, i=1, \ldots, n_{\min }^{\prime}$. Note that the dimensions of the precoding matrix and transmit power allocation are reduced by a factor of $\left(L \times n_{\text {min }}^{\prime}\right) /\left(n_{\mathrm{t}} \times n_{\text {min }}\right)$ and $n_{\min }^{\prime} / n_{\min }$, respectively, implying smaller feedback overhead in the proposed scheme. The capacity of the proposed system is given by

$$
C_{\text {part }}=\log _{2} \operatorname{det}\left(\mathbf{I}+\mathbf{H}^{\prime} \mathbf{K}_{x_{L}} \mathbf{H}^{\prime *}\right)=\sum_{i=1}^{n_{\min }^{\prime}} \log _{2}\left(1+\lambda_{i}^{\prime 2} P_{i}^{\prime}\right) .
$$

\section{Performance Analysis}

We analyze the performance in terms of the capacity loss defined by

$$
\begin{aligned}
\Delta & \triangleq C_{\text {full }}-C_{\text {part }} \\
& =\sum_{i=1}^{n_{\min }} \log _{2}\left(1+\lambda_{i}^{2} P_{i}\right)-\sum_{i=1}^{n_{\min }^{\prime}} \log _{2}\left(1+\lambda_{i}^{\prime 2} P_{i}^{\prime}\right) .
\end{aligned}
$$

\section{A. High SNR Environments}

In high SNR environments, the optimum power allocation is to earallv assign the power to each spatial channel, i.e., $P_{i}^{\prime}=P / n_{\min }^{\prime}$ for $i=1, \ldots, n_{\min }^{\prime} \quad[10]$. Then, the capacity loss can approximately be represented as

$$
\begin{aligned}
\Delta & \approx \sum_{i=1}^{n_{\min }} \log _{2}\left(\lambda_{i}^{2} \frac{P}{n_{\min }}\right)-\sum_{i=1}^{n_{\min }^{\prime}} \log _{2}\left(\lambda_{i}^{2} \frac{P}{n_{\text {min }}^{\prime}}\right) \\
& = \begin{cases}\infty, & \text { if } L<n_{\text {min }} \\
\sum_{i=1}^{n_{\min }} \log _{2}\left(\frac{\lambda_{i}^{2}}{\lambda_{i}^{\prime 2}}\right), & \text { otherwise. }\end{cases}
\end{aligned}
$$

It can be seen that the capacity loss increases to infinity when $L<n_{\min }$ mainly due to the loss of spatial multiplexing gain. This implies that the number of dominant eigen-dimensions should be chosen such that $L \geq n_{\min }$, i.e., $n_{\min }^{\prime}=n_{\min }$.

When $L \geq n_{\min }$, by letting $\lambda_{i}^{2}=\lambda_{i}^{\prime 2}+\varepsilon_{i}$, where $\varepsilon_{i}$ is a nonnegative real number, the capacity loss can be represented as

$$
\begin{aligned}
\Delta & \approx \sum_{i=1}^{n_{\min }} \log _{2}\left(1+\frac{\varepsilon_{i}}{\lambda_{i}^{\prime 2}}\right) \leq n_{\min } \log _{2}\left(1+\frac{1}{n_{\min }} \sum_{i=1}^{n_{\min }} \frac{\varepsilon_{i}}{\lambda_{i}^{\prime 2}}\right) \\
& \leq n_{\min } \log _{2}\left(1+\frac{1}{n_{\text {min }} \lambda_{\text {min }}^{\prime 2}} \sum_{i=1}^{n_{\min }} \varepsilon_{i}\right)
\end{aligned}
$$

where the first inequality is from the Jensen's inequality and the second one is from that $\lambda_{\text {min }}^{\prime}$ is the minimum singular value of $\mathbf{H}^{\prime}$. Since the sum of eigenvalues of a matrix can be expressed by its trace [9], it can be shown that

$$
\begin{aligned}
\sum_{i=1}^{n_{\min }} \varepsilon_{i} & =\sum_{i=1}^{n_{\min }} \lambda_{i}^{2}-\sum_{i=1}^{n_{\min }} \lambda_{i}^{\prime 2} \\
& =\operatorname{tr}\left[\mathbf{H} \mathbf{H}^{*}\right]-\operatorname{tr}\left[\mathbf{H}^{\prime} \mathbf{H}^{\prime *}\right] \\
& =\sum_{i=L+1}^{n_{\mathrm{t}}}\left\|\tilde{\mathbf{h}}_{i}^{\prime}\right\|^{2} \sigma_{i}^{2}
\end{aligned}
$$

where $\operatorname{tr}\left[\mathbf{A} 1\right.$ denotes the trace of matrix $\mathbf{A}$ and $\tilde{\mathbf{h}}_{i}^{\prime}$ is the $i$-th column of $\tilde{\mathbf{H}}^{\prime}(=\tilde{\mathbf{H}} \mathbf{Q})$. Note that $\tilde{\mathbf{h}}_{i}^{\prime}$ has the same distribution as $\tilde{\mathbf{h}}_{i}$. Thus, it can be shown that

$$
\Delta \leq n_{\min } \log _{2}\left(1+\frac{1}{n_{\min } \lambda_{\min }^{\prime 2}}\left(\sum_{i=L+1}^{n_{\mathrm{t}}}\left\|\tilde{\mathbf{h}}_{i}^{\prime}\right\|^{2} \sigma_{i}^{2}\right)\right) .
$$

Note that $\lambda_{\min }^{\prime 2}$ is independent of $\mathbf{h}_{i}^{\prime}, i=L, \ldots, n_{\mathrm{t}}$, since $\lambda_{\min }^{\prime 2}$ is the minimum eigenvalue of $\left[\mathbf{h}_{1}^{\prime} \cdots \mathbf{h}_{L}^{\prime}\right]$. It can be shown that the expected value of the capacity loss is bounded by

$$
\begin{aligned}
E\{\Delta\} & \leq E\left\{n_{\min } \log _{2}\left(1+\frac{1}{n_{\min } \lambda_{\min }^{\prime 2}}\left(\sum_{i=L+1}^{n_{\mathrm{t}}}\left\|\tilde{\mathbf{h}}_{i}^{\prime}\right\|^{2} \sigma_{i}^{2}\right)\right)\right\} \\
& \leq n_{\min } \log _{2}\left(1+E\left\{\frac{1}{n_{\min } \lambda_{\min }^{\prime 2}}\left(\sum_{i=L+1}^{n_{\mathrm{t}}}\left\|\tilde{\mathbf{h}}_{i}^{\prime}\right\|^{2} \sigma_{i}^{2}\right)\right\}\right) \\
& =n_{\min } \log _{2}\left(1+\frac{n_{\mathrm{r}}}{n_{\text {min }}} E\left\{\frac{1}{\lambda_{\text {min }}^{\prime 2}}\right\}\left(\sum_{i=L+1}^{n_{\mathrm{t}}} \sigma_{i}^{2}\right)\right) .
\end{aligned}
$$

It can be seen that the capacity loss is mainly affected by the amount of neglected eigenvalues of the channel correlation matrix.

\section{B. Low SNR Environments}

In low SNR environments, the capacity can be maximized by allocating all the power to the strongest spatial channel [10]. The corresponding capacity can be represented as

$$
\begin{aligned}
\Delta & \approx \log _{2}\left(1+\lambda_{\max }^{2} P\right)-\log _{2}\left(1+\lambda_{\max }^{\prime 2} P\right) \\
& \approx \lambda_{\max }^{2} P \log _{2} e-\lambda_{\max }^{\prime 2} P \log _{2} e
\end{aligned}
$$

where $\lambda_{\max }$ and $\lambda_{\max }^{\prime}$ are the maximum singular values of $\mathbf{H}$ and $\mathbf{H}^{\prime}$, respectively. Using that

$$
\lambda_{\max }^{2}-\lambda_{\max }^{22} \leq \operatorname{tr}\left[\mathbf{H} \mathbf{H}^{*}\right]-\operatorname{tr}\left[\mathbf{H}^{\prime} \mathbf{H}^{\prime *}\right]
$$

the capacity loss can be rewritten as

$$
\begin{aligned}
\Delta & \leq\left(\operatorname{tr}\left[\mathbf{H} \mathbf{H}^{*}\right]-\operatorname{tr}\left[\mathbf{H}^{\prime} \mathbf{H}^{\prime *}\right]\right) P \log _{2} e \\
& =\left(\sum_{i=L+1}^{n_{\mathrm{t}}}\left\|\tilde{\mathbf{h}}_{i}^{\prime}\right\|^{2} \sigma_{i}^{2}\right) P \log _{2} e .
\end{aligned}
$$


Finally, the expected value of the capacity loss is given by

$$
E\{\Delta\} \leq n_{\mathrm{r}}\left(\sum_{i=L+1}^{n_{t}} \sigma_{i}^{2}\right) P \log _{2} e .
$$

Note that as in the high SNR case, the performance loss is also related to the amount of neglected eigenvalues of the channel correlation matrix.

The analytic results suggest that the capacity loss of the proposed scheme can be minimized by properly choosing the number of dominant eigen-dimensions of the channel correlation matrix. As an example, it may be desirable for low SNR users to choose the smallest $L$ satisfying

$$
\sum_{i=L+1}^{n_{\mathrm{t}}} \sigma_{i}^{2} \leq \delta_{\mathrm{th}}
$$

where $\delta_{\mathrm{th}}$ is a threshold value to be optimized by considering both the feedback overhead and performance loss, and for high SNR users to choose a value of $L$ larger than $n_{\min }$ while satisfying (24).

\section{Simulation Results}

The performance of the proposed scheme is verified by computer simulation in Rayleigh fading channels. We assume that four transmit antennas and two receive antennas are equally separated by four wave lengths and half wave length in a row, respectively, unless stated otherwise. Channel model is assumed to be the suburban macro scenario of tapped delayline parameters in [11]. We also assume that the feedback information is reported without delay except Fig. 4 and without quantization except Fig. 5.

Fig. 2 depicts the performance of the proposed scheme according to the average SNR when $L=2$. It can be seen that the use of $L=2$ provides almost the same capacity as the full adaptation scheme, while reducing the dimension of the precoding matrix by one half.

Fig. 3 depicts the performance of the proposed scheme according to the change of antenna spacing between the transmit antennas, where small antenna spacing means larger correlation between the adjacent antennas. It can be seen that in low SNR environments, the proposed scheme with $L=1$ can provide performance comparable to the full adaptation scheme as the correlation increases, while significantly reducing the dimension of the precoding matrix to be reported. On the other hand, in high SNR environments, the proposed scheme with $L=1$ is not desirable mainly due to the loss of spatial multiplexing gain as indicated in (15). However, the use of $L=2$ is affordable when the channel correlation is high.

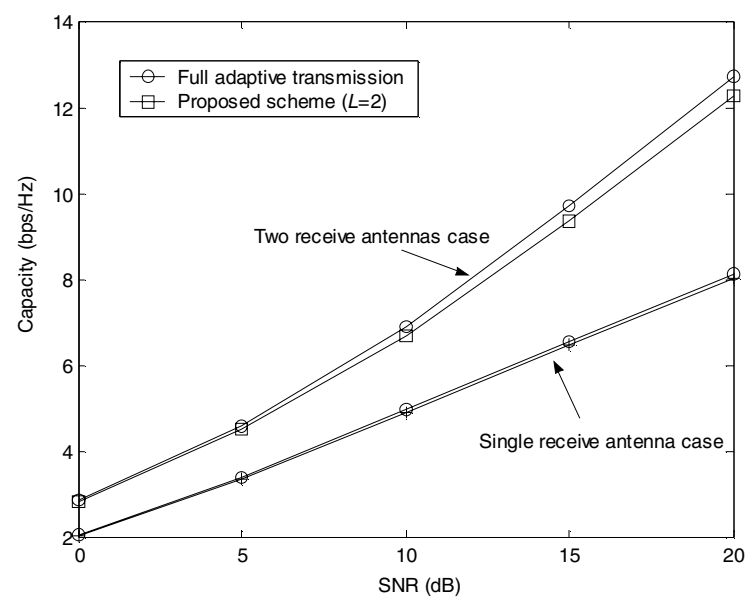

Fig. 2. Performance of the proposed scheme when $L=2$.

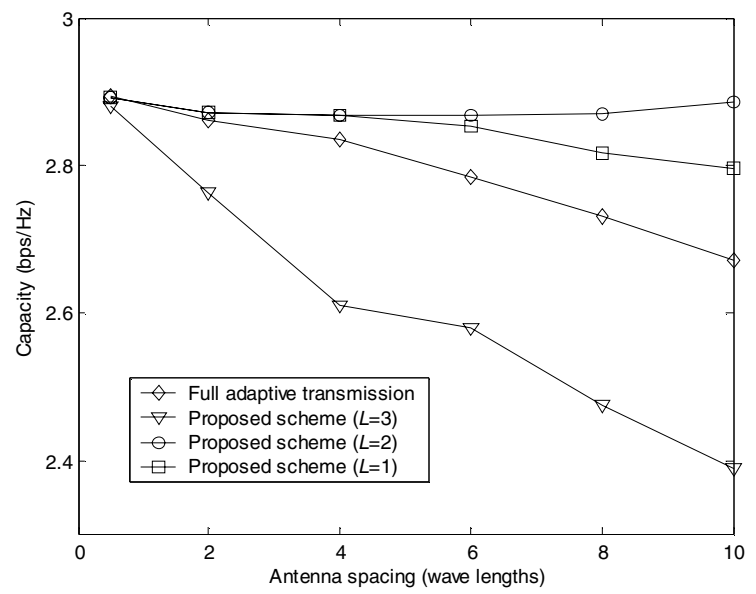

(a) When $\mathrm{SNR}=0 \mathrm{~dB}$.

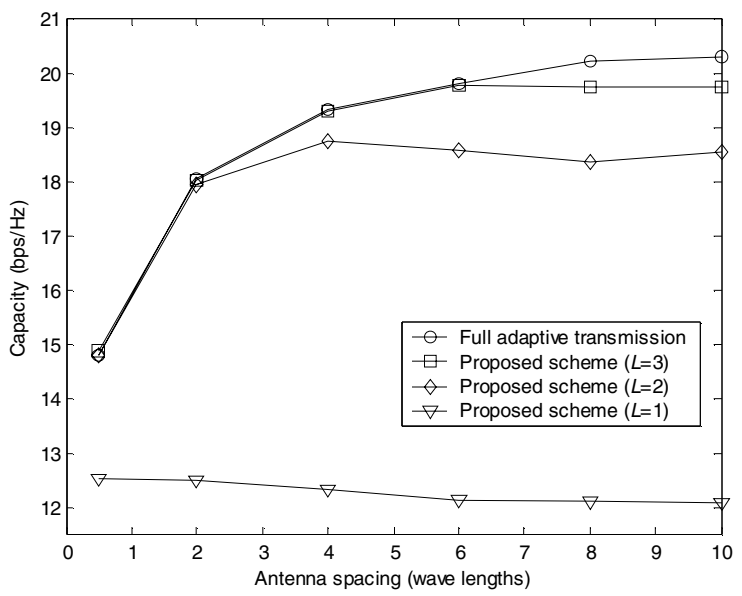

(b) When $\mathrm{SNR}=30 \mathrm{~dB}$.

Fig. 3. Performance according to the change of antenna spacing. 
Fig. 4 depicts the performance of the proposed scheme with outdated channel information. We assume that the average SNR is $10 \mathrm{~dB}$ and $L=2$. We also assume that the instantaneous channel information and the channel correlation information are reported to the transmitter at every $5 \mathrm{~ms}$ and $10000 \mathrm{~ms}$, respectively, with a time delay of $5 \mathrm{~ms}$. It can be seen that performance loss due to the outdated channel information is less in the proposed scheme. This is mainly due to the fact that the SNR gain by exploiting the correlation information is less sensitive to the user mobility. It can also be seen that the use of correlation information reported at every $10000 \mathrm{~ms}$ provides negligible performance loss compared to the use of perfect correlation information resulting in marginal feedback overhead for the long term precoding matrix $\mathbf{Q}_{L}$.

Fig. 5 depicts the performance with the use of quantized channel information when four transmit antennas and a single receive antenna is used. We assume that the average SNR is 10 $\mathrm{dB}$ and $L=2$. Since the channel rank is always one when a single receive antenna is used, all power is allocated to the principle eigenmode. Therefore, only the precoding matrix (or equivalently beam weight vector in this case) needs to be reported to the transmitter. For the reporting of beam weight, the quantized maximum ratio transmission codebook in [2] is used. It can be seen that for a given amount of feedback information, the proposed scheme outperforms the full adaptive transmission scheme. It can also be seen that the proposed scheme can achieve desirable performance with beam weight reported with 4-bit precision, but the full adaptive transmission scheme requires the beam weight reported with at least 10-bit precision.

\section{CONCLUSIONS}

We have proposed a new adaptive MIMO scheme that works with partial channel information. By considering dominant eigen-dimensions of the channel correlation matrix, the proposed scheme can exploit most of the channel gain, while significantly reducing the feedback signaling overhead. The performance of the proposed scheme has been analyzed in terms of the capacity loss and verified by computer simulation. The simulation results show the proposed scheme outperforms the full adaptive transmission scheme when the channel information is reported with a finite precision.

\section{REFERENCES}

[1] E. Telatar, "Capacity of multi-antenna Gaussian channels," Eur. Trans. Telecomm. ETT, vol. 10, no. 6, pp. 585-596, Nov. 1999.

[2] D. Love, R. Heath, Jr. and T. Strohmer, "Grassmannian beamforming for multiple-input multiple-output wireless systems," IEEE Trans. Inform. Theory, vol. 49, pp. 2735-2747, Oct. 2003.

[3] J. C. Roh and B. D. Rao, "Design and analysis of MIMO spatial multiplexing systems with quantized feedback," IEEE Trans. Sig. Proc., vol. 54, no. 8, Aug. 2006.

[4] S. Jafar and A. Goldsmith, "Transmitter optimization and optimality of beamforming for multiple antenna systems," IEEE Trans. Wireless Commun., vol. 3, no. 4, pp. 1165-1175, July 2004.
[5] A. M. Tulino, A. Lozano and S. Verdu, "Capacity-achieving input covariance for single-user multi-antenna channels," IEEE Trans. Wireless commun., vol. 5, no. 3, Mar. 2006.

[6] M. Nicoli, O. Simeone and U. Spagnolini, "Multislot estimation of fast varying communication channels," IEEE Trans. Sig. Proc., vol. 5, pp. 11841195, May 2003.

[7] A. Paulraj, R. Nabar and D. Gore, Introduction to Space-Time Wireless Communications, Cambridge University Press, 2003.

[8] J. Kermoal, L. Schumacher, K. Pedersen, P. Mogensen and F. Frederiksen, "A stochastic MIMO radio channel model with experimental validation," IEEE J. Select. Areas Commun., vol. 20, no. 6, pp. 1211-1226, Aug. 2002.

[9] G. Strang, Linear Algebra and its Applications, Third Edition, San Diego, Harcourt Brace Jovanovich, Publishers, 1988.

[10] D. Tse and P. Viswanath, Fundamentals of Wireless Communication, Cambridge University Press, 2005.

[11] D. S. Baum, J. Hansen, and J. Salo, "An interim channel model for beyond-3G systems: extending the 3GPP spatial channel model (SCM)," in Proc. IEEE Veh. Technol. Conf., vol. 5, pp. 3132-3136, May 2005.

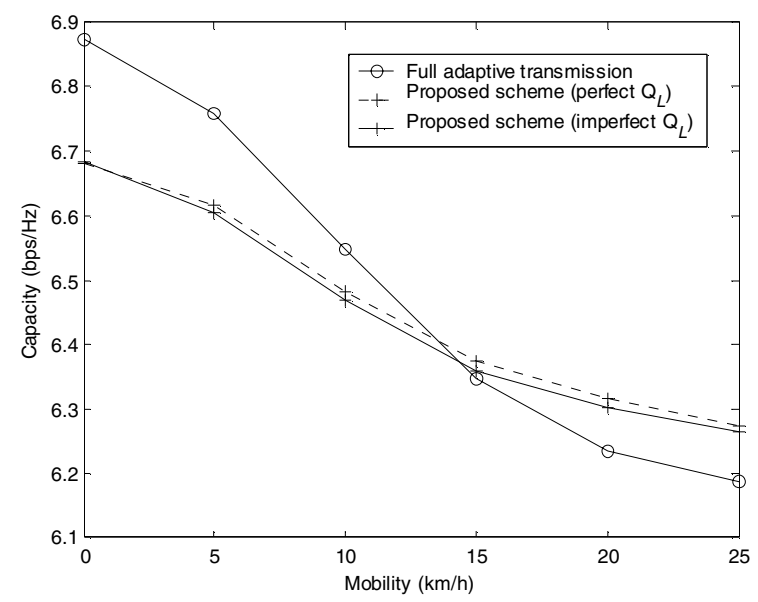

Fig. 4. Performance with outdated channel information.

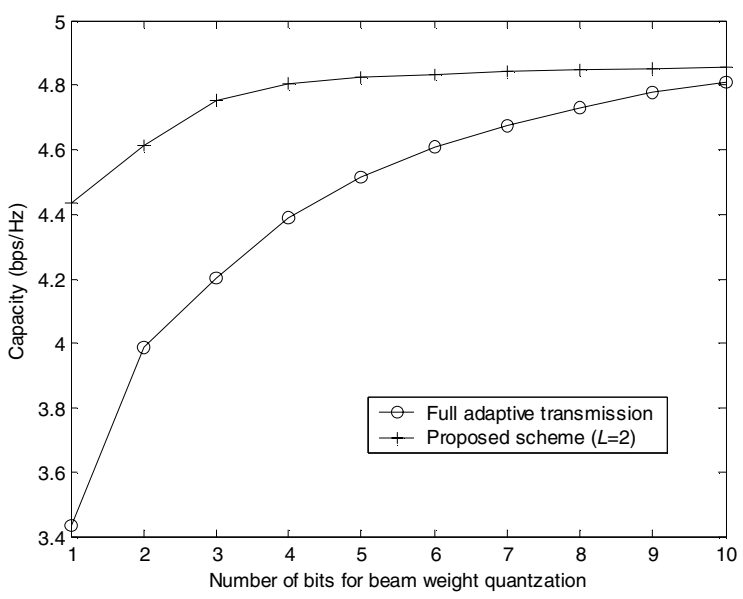

Fig. 5. Performance with quantized channel information. 